\title{
Evaluación de ocho cultivares de pimiento tipo "ancho" bajo las condiciones del Valle de Casma
}

\author{
Performance of eight ancho peppers cultivars \\ under Casma Valley conditions
}

A. Casas ${ }^{1}$; C. Saldaña ${ }^{2}$; K. Coronado ${ }^{3}$, U. Osorio ${ }^{4}$

\begin{abstract}
Resumen
El presente trabajo de investigación se realizó en el fundo "Santa Delfina" ubicado en el Valle de Casma, Ancash, con el objetivo de evaluar el comportamiento agronómico de ocho cultivares de pimiento tipo "Ancho" (Capsicum annuum L.) y determinar cuál de estos se adaptan mejor en términos de rendimiento y calidad de fruto. El diseño estadístico utilizado fue bloques completos al azar con ocho tratamientos y cuatro repeticiones. Hubo diferencias significativas en todas las variables evaluadas. Se concluye que los cultivares que presentaron mayor precocidad fueron Amazonas, Limeño y Máncora, con 20 días de ventaja en la cosecha. Los mejores cultivares para el rendimiento de frutos frescos por hectárea fueron Huizache, San Juan y Caballero con 55,71 ton/ha, 51,99 ton/ha y 51,71 ton/ha, respectivamente. Los que presentaron el mayor rendimiento comercial en seco fueron los cultivares Huizache, San Juan y Capulín con 10,77 ton/ ha, 10,66 ton/ha y 10,18 ton/ha, respectivamente. El cv. Máncora produjo los frutos más largos en fresco y seco (14,3 cm y $14,23 \mathrm{~cm}$, respectivamente). El cv. Capulín produjo los frutos más anchos en fresco $(7,85 \mathrm{~cm})$ y seco $(7,6 \mathrm{~cm})$. El mayor número de frutos por planta lo obtuvieron Huizache $(16,2)$, Caballero $(13,37)$ y San Juan $(13,06)$. Los frutos frescos y secos más pesados lo produjeron Máncora (124,99 gr) y Tiburón (18,23 gr), respectivamente. La mejor relación peso fresco/peso seco (PF/PS) lo tuvieron Capulín $(4,9)$, San Juan $(4,8)$ y Tiburón $(5,01)$.
\end{abstract}

Palabras clave: calidad; Capsicum annuит L.; chile ancho; pimiento; rendimiento.

\begin{abstract}
A trial was conducted in the "Santa Delfina" farm located in Casma, Ancash, to evaluate the agronomic performance of eight "Ancho" pepper cultivars (Capsicum annuum L.) and determine which of these could adapt better in terms of yield and fruit quality. A randomized complete block design was used with eight treatments and four replications. There were significant differences in all the cultivars evaluated. The cultivars Amazonas, Máncora and Limeño were twenty days earlier compared to the others cultivars. The best cultivars for fresh fruit yield/ha were Huizache, San Juan and Caballero (55,71 ton/ha, 51,99 ton/ha and 51,71 ton/ha, respectively). Huizache, San Juan and Capulín had the best dry fruit yield/ ha $(10,77$ ton/ha, 10,66 ton/ha and 10,18 ton/ha, respectively). Máncora presented the longest fresh and dry fruits (14,3 $\mathrm{cm}, 14,23 \mathrm{~cm})$. Capulín presented the widest fresh and dry fruits $(7,85 \mathrm{~cm}, 7,6 \mathrm{~cm})$. The cultivars that presented more of fruits/plant were Huizache $(16,2)$, Caballero $(13,37)$, and San Juan $(13,03)$. The heaviest fresh and dry fruits were observed in Máncora (124,99 gr) and Tiburón (18,23 gr), respectively. The best fresh weight/dry weight rate (FW/DW) was observed in Capulín (4,59), San Juan $(4,83)$ and Tiburón $(5,02)$.
\end{abstract}

Keywords: quality; Capsicum annuum L.; chile ancho; pepper; yield.

\section{Introducción}

El pimiento es uno de los productos de exportación hortícola de gran relevancia en el Perú. Dentro de este grupo destaca la páprika, que se utiliza como colorante natural en las industrias de alimentos, cosméticos y farmacéutica. Asimismo, el pimiento tipo "Ancho", que se siembra mucho en México y cuyo fruto es similar al pimiento morrón, pero un poco más largo, es un producto de agro exportación en pleno apogeo en la costa de nuestro país; es un fruto no perecible y se exporta deshidratado para su uso en la cocina; es ideal para salsas, adobos y diversos guisos. Por tanto, es imprescindible la adquisición de cultivares superiores con la finalidad de darle un potencial a este nuevo pimiento que se cataloga como uno de los más importantes Capsicum deshidratados que es consumido en 
los Estados Unidos, principalmente por personas de origen mexicano.

En el 2010, nuestro país exportó US\$ 50,09 millones en valor FOB de pimiento piquillo en conserva. Las exportaciones de páprika y otros pimientos secos alcanzaron los US\$ 11,9 millones (4,547 TM) en valor FOB, 17,6 \% más que en el periodo anterior. De esta forma, los Capsicum son considerados como la segunda hortaliza de exportación, sólo detrás del espárrago (SUNAT, 2011).

En el Perú, a pesar de que los productores de las diferentes localidades emplean ciertos paquetes tecnológicos para la explotación del cultivo de pimiento, el aporte para este rubro sigue en desarrollo, ya que la extrema fragmentación de la propiedad agrícola y su gran dispersión constituye un límite a la eficiencia productiva y a la consolidación de la articulación entre los compradores y los proveedores. Esto puede ser mitigado si se utilizan cultivares apropiados acordes con las condiciones agro ecológicas de la zona. Por tal motivo, es muy importante realizar experimentos con cultivares en diferentes años y distintas localidades y ver su potencial, antes de recomendar su uso comercial.

El presente trabajo de investigación tuvo por objetivo evaluar ocho cultivares de pimiento tipo Ancho, todos híbridos, en términos de rendimiento y calidad de fruto bajo las condiciones del valle de Casma.

\section{Materiales y métodos}

El ensayo se realizó en un suelo de textura franco arenosa, $\mathrm{pH} \mathrm{7,67} \mathrm{y} \mathrm{1,4 \%} \mathrm{de} \mathrm{materia} \mathrm{orgánica,} \mathrm{durante} \mathrm{los} \mathrm{meses} \mathrm{de}$ marzo a noviembre de 2010 en el fundo "Santa Delfina" ubicado en Casma, provincia de Ancash. La temperatura máxima se registró en abril con $33,6{ }^{\circ} \mathrm{C}$ y la mínima en agosto con $10,2^{\circ} \mathrm{C}$. Los cultivares evaluados fueron San Juan, Tiburón, Caballero, Capulín, Huizache, Amazonas, Limeño y Máncora (Tabla 1). El diseño estadístico utilizado fue el de bloques completos al azar con cuatro repeticiones. Cada unidad experimental estuvo constituida por una parcela de tres surcos de $5 \mathrm{~m}$ de longitud, distanciados a 1,2 m y con una distancia entre plantas de 0,25 m. Las semillas fueron proporcionadas directamente por las casas comerciales de semillas y las plántulas fueron preparadas en SF Almácigos de Virú, La Libertad.

Se llevó a cabo la preparación del campo un mes antes del trasplante para lo cual se utilizaron plantines de 40 días de edad. El riego fue por gravedad con aguas del río Casma; además, los riegos se complementaron con agua de pozo cuando los niveles de agua del río disminuyeron. Se realizaron las respectivas fertilizaciones en tres oportunidades llegando a un total de $263 \mathrm{~N}-128,13 \mathrm{P}-387 \mathrm{~K}-$ $83 \mathrm{Ca}-0,75 \mathrm{Mg}$ a los 9, 31, $61 \mathrm{ddt}$. Se realizaron los controles de malezas en tres oportunidades de manera manual (repique) y con aplicaciones de herbicida (glifosato) en forma dirigida y con campana.

Alos 15 días del trasplante (ddt) se iniciaron las evaluaciones de las variables. El inicio de floración fue considerado tan pronto se presentó una flor en una planta, el $100 \%$ de floración se consideró cuando el total de plantas presentaba flor; el inicio de la coloración se determinaba cuando un fruto presentaba las primeras señales de color rojo, el 100 $\%$ de coloración se consideraba cuando todos los frutos presentaban inicio de coloración roja en los frutos; el inicio de cosecha se consideraba cuando los frutos mostraban inicio de deshidratación. Se cosechó la hilera central eliminando las plantas de los extremos para disminuir el efecto de borde. Se evaluaron las variables de precocidad de floración, pintado y cosecha (inicio e uniformidad), rendimiento comercial en fresco y seco, el secado fue al medio ambiente con túneles de plástico, calidad de fruto fresco y seco (ancho, longitud y peso, determinados en una muestra al azar de 10 frutos en cada cosecha), número de frutos/planta, que se determinó dividiendo el número total de frutos producidos por el número de plantas en cada parcela y relación peso fresco/peso seco del fruto(PF/PS). Se realizaron cinco cosechas, la primera a los $125 \mathrm{ddt} y$ la última a los $255 \mathrm{ddt}$, en el punto de cosecha (inicios de deshidratación), de manera independiente para cada cultivar. Se realizó el análisis de variancia y el comparativo de medias con la prueba de Duncan al 0,05.

\section{Resultados y discusión}

\section{Inicio de la floración}

El cv. Limeño fue el primero en iniciar la floración a los 16 días después del trasplante (ddt), seguido de los cultivares Máncora a los 19 ddt; Amazonas y San Juan a los 20 ddt; Tiburón, Caballero y Huizache a los 22 ddt y finalmente Capulín a los 23 ddt (Tabla 2). Esta

Tabla 1. Cultivares de pimiento tipo "Ancho" evaluados en Casma

\begin{tabular}{|l|l|l|l|l|}
\hline Tratamiento & Cultivar & Tipo & Distribuidor & Procedencia \\
\hline 1 & San Juan & Rojo & Seminis Seed & Estados Unidos \\
\hline 2 & Tiburón & Rojo & Sakata & Japón \\
\hline 3 & Caballero & Rojo & Sakata & Japón \\
\hline 4 & Capulín & Mulato & Harris Morán & Estados Unidos \\
\hline 5 & Huizache & Rojo & Harris Morán & Estados Unidos \\
\hline 6 & Amazonas & Rojo & Semillas del Mundo & Estados Unidos \\
\hline 7 & Limeño & Rojo & Semillas del Mundo & Estados Unidos \\
\hline 8 & Máncora & Rojo & Semillas del Mundo & Estados Unidos \\
\hline
\end{tabular}


característica es muy propia de cada cultivar, por lo observado en los datos obtenidos la floración se inicia entre los 16 y $23 \mathrm{ddt}$ bajo las condiciones del valle de Casma.

\section{Floración total}

El cv. Limeño fue el que logró la uniformidad de floración de forma temprana (a los $38 \mathrm{ddt}$ ), los cultivares Máncora y Amazonas lo hicieron a los $40 \mathrm{ddt}$; los cultivares San Juan, Huizache y Capulín uniformizaron floración a los 42 ddt y finalmente los cv. Tiburón y Caballero uniformizaron su floración a los 45 ddt (Tabla 2). Se puede concluir que el cultivar Limeño es el más temprano en su proceso de floración y el cultivar Tiburón el que tiende a ser el más tardío, al menos bajo las condiciones de Casma.

\section{Inicio de pintado de frutos}

Una pronta coloración de frutos se percibió a los 66 ddt en el cv. Máncora, seguido cercanamente de los cv. Limeño y Amazonas a los 68 ddt y $72 \mathrm{ddt}$, respectivamente (Tabla 2). Posteriormente, los cv. San Juan, Tiburón, Caballero y Capulín iniciaron su coloración de frutos a los 77, 80, 82 y 86 ddt, respectivamente. Finalmente el cv. Huizache inició su coloración a los 90 ddt. Esta característica es importante cuando estos frutos son destinados para consumo en fresco ya que los consumidores en fresco los prefieren cuando aún obtenidos, los cultivares Amazonas, Limeño y Máncora (en orden de precocidad) tuvieron un similar comportamiento y adaptabilidad en campo, por lo cual se les considera como los cultivares más tempranos o precoces al ser comparados con los cinco materiales restantes. Por otra parte, los cultivares (ordenados del precoz al tardío) Capulín, San Juan, Tiburón, Caballero y Huizache se comportaron de manera semejante sin tener una diferencia de días distanciados entre ellos (Tabla 2).

\section{Rendimiento}

\section{Rendimiento comercial en fresco}

Los rendimientos en fresco de los ocho cultivares evaluados, se presentan en la Tabla 3 , donde se aprecia que los rendimientos varían de 19,01 ton/ha hasta 55,71 ton/ ha. El promedio general del ensayo fue de 39,68 ton/ha. La prueba de Duncan al $5 \%$ muestra que los cultivares Huizache, San Juan, Caballero, Capulín y Tiburón fueron estadísticamente superiores a los cultivares Amazonas, Limeño y Máncora (Tabla 3).

Larriaga, Camacho, Murillo y Aguilar (2004) evaluaron los híbridos Caballero y Tiburón F1 y F2 con diferentes tratamientos de fertilización bajo riego por goteo. Los mejores rendimientos fueron 61,1 ton/ha de fruto verde

Tabla 2. Floración y fructificación en ocho cultivares de pimiento (Capsicum annuum) tipo "Ancho" bajo las condiciones del valle de Casma (2010)

\begin{tabular}{|l|l|l|l|l|l|}
\hline \multicolumn{2}{|l|}{ Floración (ddt) } & \multicolumn{4}{l|}{ Fructificación (ddt)* } \\
\hline Cultivares & inicio de floración & $\mathbf{1 0 0} \%$ de floración & inicio de pintado & $\mathbf{1 0 0} \%$ de pintado & inicio de cosecha \\
\hline San Juan & 20 & 42 & 77 & 121 & 145 \\
\hline Tiburón & 22 & $\mathbf{4 5}$ & 80 & 123 & 145 \\
Caballero & 22 & $\mathbf{4 5}$ & 82 & 127 & 145 \\
Capulín & $\mathbf{2 3}$ & 42 & 86 & 118 & 145 \\
Huizache & 22 & 40 & $\mathbf{9 0}$ & $\mathbf{1 2 9}$ & 145 \\
Amazonas & 20 & 40 & 72 & $\mathbf{9 9}$ & 125 \\
Limeño & $\mathbf{1 6}$ & $\mathbf{3 8}$ & 68 & 110 & 125 \\
Máncora & 19 & 40 & $66-90$ & 104 & 125 \\
\hline RANGO & $16-23$ & $38-45$ & & $99-129$ & $125-145$ \\
\hline
\end{tabular}

"ddt: días después del trasplante.

no tienen coloración.

\section{Coloración total de los frutos}

A los 99 ddt, el cv. Amazonas logró un pintado de frutos uniforme, seguido de los cv. Máncora y Limeño a los 104 y $110 \mathrm{ddt}$, respectivamente (Tabla 2). Días después lograron uniformidad de coloración los cv. Capulín, San Juan, Tiburón y Caballero a los 118, 121, 123 y 127 ddt, respectivamente. Por último, el cv. Huizache logró uniformidad de pintado a los $129 \mathrm{ddt}$.

\section{Inicio de cosecha}

Los cultivares que lograron temprano el punto de cosecha para secado de fruto fueron el cv. Limeño, Máncora y Amazonasa a los 125 ddt. El resto de cultivares cv. San Juan, Tiburón, Capulín, Caballero y Huizache coincidieron en el punto de cosecha a los 145 ddt, alcanzándolo en la primera cosecha, 20 días después. De los resultados para el híbrido Caballero, con el tratamiento consistente en 400 kilos de nitrógeno y riegos por 2,5 horas/día, mientras que para el híbrido Tiburón F1 y F2 produjeron 40,6 y 39,2 ton/ha de fruto verde con el mismo tratamiento de 400 kilos de nitrógeno/ha y riegos por ocho horas cada tercer día. Los rendimientos bajo las condiciones de Casma fueron similares con 51,71 ton/ha para el cv. Caballero y 46,57 ton/ha para el cv. Tiburón, ambos procedentes de la firma japonesa Sakata.

Otro estudio realizado y publicado por el Instituto Nacional de Investigaciones Forestales, Agrícolas y Pecuarias - INIFAP Zacatecas en el 2006, evaluó al híbrido Caballero bajo las condiciones climáticas del Altiplano de Zacatecas, donde dicho material mostró adaptabilidad y buen potencial, con un rendimiento de 55,8 ton/ha de fruto verde para ser consumido como verdura, frente a otros 12 
híbridos. Menciona de igual manera al híbrido Tiburón con 50,00 ton/ha, ubicándose en el 7mo lugar según el potencial de rendimiento mostrado.

En el estudio mencionado, cabe resaltar que los cultivares Caballero y Tiburón muestran un importante potencial frente a condiciones no tan idóneas y recomendadas para los Capsicum, ya que Zacatecas posee un clima seco, con una temperatura media anual mayor a $18^{\circ} \mathrm{C}$ y presencia de lluvias y heladas ocasionales. Pese a esto, ambos presentaron considerables rendimientos y el cv. Caballero, en reiteradas ocasiones, fue superior al cv. Tiburón. Esto mismo se reafirma para las condiciones de Casma, en donde se hallan adecuadas condiciones climáticas para dicho cultivo. Se obtuvo un mayor potencial de rendimiento con 51,71 ton/ha por parte del cv. Caballero frente a 46,56 ton/ ha del cv. Tiburón.

Ramos-Gourcy y De Luna-Jiménez (2006) compararon los cultivares San Juan y Caballero con un cultivar tradicional

Tabla 3. Rendimiento fresco y seco (ton/ha) de ocho cultivares de pimiento tipo "Ancho" (Capsicum annuun L).

\begin{tabular}{|l|c|c|}
\hline \multirow{2}{*}{ Cultivares } & \multicolumn{2}{|c|}{ Rendimiento (ton/ha) } \\
\cline { 2 - 3 } & Fresco & Seco \\
\hline Huizache & $55,71 \mathrm{a}$ & $10,76 \mathrm{a}^{*}$ \\
\hline San Juan & $51,99 \mathrm{a}$ & $10,65 \mathrm{a}$ \\
\hline Caballero & $51,71 \mathrm{a}$ & $9,44 \mathrm{a}$ \\
\hline Capulín & $46,82 \mathrm{a}$ & $10,18 \mathrm{a}$ \\
\hline Tiburón & $46,57 \mathrm{a}$ & $9,36 \mathrm{a}$ \\
\hline Amazonas & $23,1 \mathrm{~b}$ & $3,40 \mathrm{~b}$ \\
\hline Limeño & $22,53 \mathrm{~b}$ & $3,22 \mathrm{~b}$ \\
\hline Máncora & $19,01 \mathrm{~b}$ & $2,43 \mathrm{~b}$ \\
\hline Promedio & 39,68 & 7,43 \\
\hline C.V. & $21,73 \%$ & $21,92 \%$ \\
\hline
\end{tabular}

estadísticas significativas, según la prueba de Duncan al $5 \%$. de los agricultores, en cuatro concentraciones de una solución hidropónica bajo invernadero, encontrando que el cv. San Juan obtuvo el mayor rendimiento con 70 ton/ha frente al cv. Caballero que arrojó 66 ton/ha; demostrando una vez más las virtudes del cv. San Juan frente a otros cultivares.

De igual manera, Santoyo, Martínez y Garzón (2009) evaluaron diferentes tipos de chiles, entre ellos el cv. Caballero, y reportaron un rendimiento de 45,4 ton/ha para este cultivar, valor similar a los reportados en estudios mencionados anteriormente.

\section{Rendimiento comercial en seco}

Los rendimientos en seco de los ocho cultivares evaluados variaron de 2,43 ton/ha hasta 10,77 ton/ha (Tabla 3). El promedio general fue de 7,43 ton/ha. Como en el caso del rendimiento en fresco, se vuelve a observar superioridad estadística, según la prueba de Duncan al $5 \%$, de los cultivares Huizache, San Juan, Capulín, Caballero y Tiburón sobre los cultivares Amazonas, Limeño y Máncora.

\section{Calidad de fruto}

\section{Ancho de fruto}

Esta variable para fruto fresco osciló entre $6,25 \mathrm{~cm} \mathrm{y}$ $7,85 \mathrm{~cm}$. El promedio general fue de $7,1 \mathrm{~cm}$. Para el fruto seco osciló entre $6,25 \mathrm{~cm}$ y $7,6 \mathrm{~cm}$. El promedio general fue de 7,01 cm (Tabla 4).

El cv. Capulín mostró el mejor valor mientras que el cv. Amazonas presentó el menor valor para ambos casos (fresco y seco). Según la prueba de Duncan al $5 \%$, los cultivares Capulín, Tiburón y San Juan fueron superiores, estadísticamente, a los cultivares Caballero y Amazonas en estado fresco y seco para esta característica. En general, se puede mencionar que no hubo mucha variación en las medidas entre frutos frescos y secos.

\section{Largo de fruto}

El fruto fresco más largo se observó en el cultivar Máncora $(14,3 \mathrm{~cm})$ y el fruto más pequeño se presentó en el cultivar Huizache $(9,85 \mathrm{~cm})$. En cuanto a la longitud del fruto, los cultivares Máncora y Limeño fueron superiores, estadísticamente, según la prueba de Duncan al $5 \%$, al

Tabla 4. Peso (gr), longitud $(\mathrm{cm})$ y ancho $(\mathrm{cm})$ promedio en frutos frescos y secos de ocho cultivares de pimiento tipo "Ancho" (Capsicum annuum L.)

\begin{tabular}{lllllll}
\hline \multirow{2}{*}{ Cultivares } & \multicolumn{2}{l}{ Peso promedio (gr) } & \multicolumn{2}{l}{ Longitud (cm) } & \multicolumn{2}{l}{ Ancho (cm) } \\
\cline { 2 - 8 } San Juan & Fresco & Seco & Fresco & Seco & Fresco & Seco \\
Tiburón & $109,57 \mathrm{abc}$ & $14,30 \mathrm{ad}$ & $12,05 \mathrm{bc}$ & $12,53 \mathrm{~b}$ & $7,43 \mathrm{ab}$ & $7,45 \mathrm{a}^{*}$ \\
Caballero & $96,86 \mathrm{bc}$ & $17,38 \mathrm{a}$ & $10,05 \mathrm{~d}$ & $9,80 \mathrm{~d}$ & $7,45 \mathrm{ab}$ & $7,15 \mathrm{ab}$ \\
Capulín & $98,50 \mathrm{bc}$ & $15,73 \mathrm{bc}$ & $10,85 \mathrm{~cd}$ & $10,95 \mathrm{~cd}$ & $6,58 \mathrm{dc}$ & $6,58 \mathrm{dc}$ \\
Huizache & $86,89 \mathrm{c}$ & $13,28 \mathrm{~d}$ & $9,85 \mathrm{~d}$ & $11,83 \mathrm{bc}$ & $7,85 \mathrm{a}$ & $7,60 \mathrm{a}$ \\
Amazonas & $122,92 \mathrm{ab}$ & $12,05 \mathrm{~d}$ & $13,00 \mathrm{bc}$ & $13,00 \mathrm{ab}$ & $7,05 \mathrm{abc}$ & $7,05 \mathrm{ab}$ \\
Limeño & $101,51 \mathrm{abc}$ & $12,05 \mathrm{~d}$ & $14,00 \mathrm{a}$ & $14,05 \mathrm{a}$ & $6,83 \mathrm{abc}$ & $6,25 \mathrm{c}$ \\
Máncora & $124,99 \mathrm{a}$ & $13,08 \mathrm{~d}$ & $14,30 \mathrm{a}$ & $14,23 \mathrm{a}$ & $7,35 \mathrm{abc}$ & $7,13 \mathrm{ab}$ \\
Promedio & 105,15 & 14,38 & 11,98 & 12,03 & 7,10 & 7,01 \\
\multicolumn{1}{c}{ C.V. } & $15,34 \%$ & $9,77 \%$ & $7,06 \%$ & $7,56 \%$ & $7,06 \%$ & $6,98 \%$ \\
\hline
\end{tabular}

(*) Medias seguidas en la misma letra no tienen diferencias estadísticas significativas, según la prueba de Duncan al $5 \%$. 
resto de cultivares (Tabla 4). El fruto seco más largo, como en el caso del fresco, se vuelve a observar en el cultivar Máncora (14,23cm), mientras que el fruto más pequeño fue del cultivar Tiburón $(9,8 \mathrm{~cm})$. Según la prueba de Duncan al $5 \%$, los cultivares Máncora, Limeño y Amazonas fueron superiores, estadísticamente, a los cultivares Tiburón y Huizache en ambos estados del fruto para la característica de la longitud. Este aspecto tuvo una tendencia promedio que tiende a incrementarse cuando el fruto se seca.

Peso del fruto. gr y 124,99 gr (Tabla 4). El promedio general fue de 105,15 gr. En estado seco, el peso varió entre 12,05 gr y 18,23 gr. El promedio general fue de 14,38 gr.

El cv. Máncora mostró el mayor valor en peso fresco en comparación con el cv. Huizache. Según la prueba de Duncan al $5 \%$, el cultivar Máncora fue estadísticamente superior al Huizache para esta característica en fresco. Para el caso de seco, sobresalen los cultivares Tiburón, Caballero y Capulín que resultan ser superiores estadísticamente a los cultivares Amazonas, Limeño, Máncora y Huizache en esta característica. Los sembríos que mostraron valores inferiores, probablemente, se deba a que no manifestaron su potencial genético frente a las condiciones tanto climáticas como edáficas donde se desarrolló el experimento, por lo que produjo frutos de menor peso.

Relacionando los pesos frescos y secos de los frutos, se muestra que no necesariamente un fruto más pesado nos dará un peso seco de mayor valor, como se observa en los cultivares Máncora y Amazonas, por ejemplo. Esto es muy importante sobre todo si el producto se comercializa mayormente en seco. Esto nos indica que muchos frutos presentan un mayor contenido de agua que otros.

\section{Número de frutos por planta}

En la Tabla 5 se resume el número de frutos por planta obtenidos de los ocho sembríos evaluados. El cultivar que presentó mayor número de frutos por planta fue el Huizache $(16,20)$ y el que obtuvo un menor número de frutos por

Tabla 5. Número de frutos/planta de ocho cultivares de pimiento tipo "Ancho" (Capsicum annuum L.).

\begin{tabular}{|l|c|}
\hline \multicolumn{1}{|c|}{ Cultivares } & Promedios \\
\hline Huizache & $16,20 \mathrm{a}^{*}$ \\
\hline Caballero & $13,37 \mathrm{ab}$ \\
\hline San Juan & $13,06 \mathrm{ab}$ \\
\hline Capulín & $11,87 \mathrm{~b}$ \\
\hline Tiburón & $11,12 \mathrm{~b}$ \\
\hline Limeño & $5,65 \mathrm{c}$ \\
\hline Amazonas & $4,76 \mathrm{c}$ \\
\hline Máncora & $3,85 \mathrm{c}$ \\
\hline Promedio & 9,98 \\
\hline C.V. & $6,98 \%$ \\
\hline
\end{tabular}

(*) Medias seguidas en la misma letra no tienen diferencias estadísticas significativas, según la prueba de Duncan al $5 \%$. planta fue el cv. Máncora $(3,85)$. En esta característica los cultivares Huizache y Caballero fueron superiores, estadísticamente, según la prueba de Duncan al $5 \%$ sobre los cultivares Amazonas y Máncora.

Ramos-Gourcy y De Luna-Jiménez (2011) evaluaron soluciones hidropónicas con los cultivares San Juan y Caballero con un tipo Criollo, bajo condiciones de invernadero. Concluyeron que el genotipo "San Juan" tuvo en promedio mayor producción de frutos por planta, seguido del híbrido "Caballero" y del Criollo. Considerando la interacción concentración*Genotipos, el híbrido Caballero, a la concentración $100 \%$ de la solución hidropónica, presentó mayor producción de frutos por planta, seguido del híbrido "San Juan" a una concentración del $100 \%$.

\section{Relación peso fresco/peso seco (PF/PS)}

La relación entre el peso del fruto fresco y el del fruto deshidratado nos indica si el fruto del pimiento maduro contiene una cantidad considerable de agua, siendo lo deseable que esta relación sea lo más baja posible (Zapata, Bañon y Cabrera, 1992).

En la Tabla 6 se resumen los valores obtenidos. Se puede apreciar que los cultivares Máncora, Limeño y Amazonas presentaron los valores más altos, superiores estadísticamente, según la prueba de Duncan al $5 \%$, al resto de cultivares. Lo cual nos indica que estos tres cultivares fueron los que presentaron mayor contenido de agua al momento de cosecha. Una relación tan alta no es favorable para el productor ya que esto nos indica que se pierde mucho peso. En cambio, los otros cultivares, que presentaron una menor relación, son los deseados por los agricultores. En esta característica sobresale el cultivar Capulín con la más baja relación, similar estadísticamente a los cultivares Tiburón y San Juan.

INIFAP Zacatecas (2006) menciona que en los campos experimentales de Zacatecas, el híbrido Caballero, bajo un

Tabla 6. Relación peso fresco/ peso seco (PF/PS) de ocho cultivares de pimiento tipo "Ancho" (Capsicum annuum L.)

\begin{tabular}{|l|c|}
\hline Cultivares & Promedios \\
\hline Máncora & $7,85 \mathrm{a}^{*}$ \\
\hline Limeño & $6,98 \mathrm{~b}$ \\
\hline Amazonas & $6,77 \mathrm{~b}$ \\
\hline Caballero & $5,46 \mathrm{c}$ \\
\hline Huizache & $5,20 \mathrm{~cd}$ \\
\hline Tiburón & $5,02 \mathrm{cde}$ \\
\hline San Juan & $4,83 \mathrm{de}$ \\
\hline Capulín & $4,59 \mathrm{e}$ \\
\hline Promedio & 5,84 \\
\hline C.V. & $5,99 \%$ \\
\hline
\end{tabular}

(*) Medias seguidas en la misma letra no tienen diferencias estadísticas significativas, según la prueba de Duncan al $5 \%$. 
buen manejo agronómico, requiere de ocho a diez kilos de fruto fresco rojo para obtener un kilo de fruto seco (PF/PS $=8$ a 10), al ser comparado con otros materiales criollos. Para el presente ensayo, el mismo material tuvo una mejor relación PF/PS, observándose que se necesita 5,45 kilos de fruto fresco para obtener un kilo de fruto seco. Estos resultados pueden deberse a las condiciones ambientales presentes durante el ensayo en la localidad de Casma, así como también al manejo agronómico.

De los resultados encontrados, los frutos del cultivar Capulín presentaron mayor materia seca y menor contenido de agua en estado fresco, ya que se necesitan cinco kilos de fruto frescos para obtener un kilo de fruto seco. Una mayor cantidad se necesita para el cultivar Máncora, ya que se debe tener cerca de ocho kilos de fruto fresco para conseguir un kilo de fruto seco; lo que hace que el cultivar Capulín sea el material con mejor relación PF/PS para fines de obtención de fruto en seco, aunque este cultivar es similar estadísticamente a los cultivares San Juan y Tiburón. Cabe mencionar que no se determinó el contenido de humedad en los frutos tanto en fresco como en seco. El criterio comercial que se usa para determinar cuando el fruto ya se considera seco, en el caso de este pimiento ancho, es cuando este parece de plástico, es decir no se rompe con facilidad y se muestra muy flexible al doblarlo o estrujarlo.

\section{Conclusiones}

Los cultivares precoces fueron Amazonas, Limeño y Máncora con una diferencia de 20 días del resto de cultivares, iniciando cosecha a los $125 \mathrm{ddt}$. Los mayores rendimientos de fruto fresco y seco se obtuvieron con los cultivares Huizache con 55,71 ton/ha y 10,77 ton/ha y San Juan con 51,99 ton/ha y 10,66 ton/ha, respectivamente, pero sin diferencia estadística entre ellos. Los frutos frescos más pesados se obtuvieron con el cultivar Máncora (124,99 gr) y los frutos frescos de menor peso con el cv. Huizache (86,89 gr), mientras que los frutos secos de mayor peso se observaron en los cultivares Tiburón y Caballero con 18,23 gr y 17,37 gr, respectivamente, sin diferencia estadística entre ellos. Los frutos secos de menor peso fueron producidos por los cultivares Limeño y Amazonas, ambos con frutos de 12,05 gr, sin guardar diferencia estadística entre sí. En cuanto a la calidad de frutos, la mayor longitud en fruto fresco lo presentó el cultivar Máncora con $14,3 \mathrm{~cm}$ de largo, mientras el de menor longitud de fruto fresco se observó en el cultivar Huizache con 9,85cm de largo de fruto. En cambio, el mayor ancho de fruto fresco lo mostró el cultivar Capulín con $7,85 \mathrm{~cm}$, en tanto el Amazonas presentó el menor ancho con $6,25 \mathrm{~cm}$. La mayor longitud en fruto seco lo mostró el cultivar Máncora, con 14,22 cm de largo de fruto, y la menor longitud de frutos, con 9,8 $\mathrm{cm}$, lo mostró el cultivar Tiburón. En cuanto al ancho de fruto seco, el mayor valor se observó en el cultivar Capulín con 7,6 cm, mientras el cultivar Amazonas presentó el valor más bajo, con $6,25 \mathrm{~cm}$. El mayor número de frutos/ planta se obtuvo con los cultivares Huizache con 16,20 y Caballero con 13,37, respectivamente, en cambio la menor relación PF/PS se observó en los cultivares Capulín y San Juan con 4,59 y 4,82, respectivamente, sin guardar diferencia estadística entre ellos. La mayor relación $\mathrm{PF} /$ PS se encontró en los cultivares Máncora y Limeño, con una relación de 7,8 y 6,9, con diferencia estadística entre ellos. Los cultivares San Juan, Tiburón, Caballero, Capulín y Huizache mostraron buen comportamiento para producción de fruto seco. Al contrario, los cultivares Amazonas, Limeño y Máncora se observan más adaptados para producción en fresco.

\section{Literatura citada}

Instituto Nacional de Investigaciones Forestales, Agrícolas y Pecuarias (INIFAP). 2006. Centro de Investigación Regional Norte Centro, Campo experimental Zacateca. Tecnología de Producción de Chile Seco. Libro Técnico $\mathrm{N}^{\mathrm{o}}$ 5. México: INIFAP/Produce. Recuperado de http://www.zacatecas.inifap.gob.mx/publicaciones/ Tecnología_de_produccion_de_chile_seco.pdf\#page $=11$

Larriaga, M.J.A.; Camacho, P.; Murillo, A. y Aguilar, G. 2004. Efecto del riego y fertilización en chiles anchos (híbridos y estándares) para zonas áridas. En: Primera Convención Mundial del Chile, pp. 206-226. León Guanajuato, México.

Santoyo, J.; Martínez, A. y Garzón, C. 2009. Validación del Potencial Productivo de Chiles Anchos y Picosos en el Sur de Sinaloa. Sinaloa, México: Fundación Produce Sinaloa, A.C. Colección de Resultados de Proyectos.

Ramos-Gourcy, F. y De Luna-Jiménez, A. 2006. Evaluación de tres Variedades de Chile (Capsicum annuum L.) en cuatro concentraciones de una solución hidropónica bajo invernadero. Diponible en: http:// www.uaa.mx/investigacion/revista/archivo/revista34/ REVISTA\%2034.pdf

Superintendencia Nacional de Administración Tributaria (SUNAT). 2011. Principales 500 Productos No Tradicionales 2009 - 2010. Diponible en: http:// www.aduanet.gob.pe/aduanas/informae/2010/exportacion/ principalesProdNoTradicionales.html

Zapata, M.; Bañón, S. y Cabrera, P. 1992. El pimiento para pimentón. (1ra. edición). Madrid, España: Ed. MundiPrensa. 